\title{
Pleuro-pulmonary ultrasound in the diagnosis and follow-up of lung infections in children with cancer: a pilot sudy
}

\author{
Mariaclaudia Meli ${ }^{1}$, Milena La Spina ${ }^{1}$, Gian Luca Trobia ${ }^{2}$, Luca Lo Nigro ${ }^{1}$, Giovanna \\ Russo $^{3}$, and Andrea Di Cataldo ${ }^{3}$ \\ ${ }^{1}$ University of Catania School of Medicine \\ ${ }^{2}$ Azienda Ospedaliera Cannizzaro \\ ${ }^{3}$ University of Catania
}

June 4, 2021

\begin{abstract}
Febrile neutropenia and lung infections are common and potential fatal complications of pediatric cancer patients during chemotherapy. Lung ultrasound (LUS) has a good accurancy in the diagnosis of pneumonia in childhood. To our knowledge there are no studies concerning its utilization in the diagnosis and follow-up of pulmonary infection in children with cancer. The goal of this pilot study is to determine the accuracy of lung ultrasonography for the diagnosis and follow up of pneumonia in children and adolescents with cancer during fever. This is a prospective observational case-control monocentric study conducted in the Pediatric Hematology and Oncology Department of University Hospital of Catania in patients aged $<18$ years with cancer, with or without fever. Attending Physician used ultrasonography to diagnose pneumonia in children with cancer during fever. Non infected cancer patients were also tested with LUS to evaluate its accuracy. When performed, the results of chest X-ray and chest CT scan were compared with LUS results. Thirty-eight patients were studied. All underwent LUS, 16 underwent CXR, 3 chest CT. Statistical analysis showed LUS specificity of $95 \%$, and sensitivity of $100 \%$; CXR, instead showed a specificity of $80 \%$ and a sensitivity of $50 \%$. The positive predictive value of LUS was $90 \%$ while that of CXR was $50 \%$. The negative predictive value of LUS was $100 \%$ while that of CXR was $80 \%$. This study shows for the first time that LUS allows physicians to diagnose pneumonia in children and young adults with cancer, with high specificity and sensitivity.
\end{abstract}

\section{Introduction}

Lung ultrasound (LUS) has been proposed as an alternative first-line imaging modality to diagnose community acquired pneumonia in children, with promising results. (1) There is evidence that LUS may have greater sensitivity, similar specificity and better inter-operator reliability in the diagnosis of pneumonia when compared with chest radiography (CXR). (2-4) Moreover, LUS is radiation free and is subject to fewer regulatory requirements, has lower cost and easier bedside availability than CXR.

LUS has also been shown to be more sensitive than chest x-ray in identifying sub-centimeter air bronchograms often not evident on CXR. (5)

Consolidations appear at LUS as hypoechoic areas. $(6,7)$ The pleural line in the wall above the consolidation is less echogenic or even disappeared. At the rear, the presence of compact vertical artifacts is frequent expression of wall reinforcements typically produced by areas with fluid content. (8,9) Interstitial pneumonia is represented by B lines isolated or confluent until to configure a white lung pattern based on gravity.

The incidence of pneumonia in cancer patients varies from 17 to $24 \%$ and clinical response to specific treatment varies from 60 to $65 \%$ with an infection-related mortality of $38 \%$. (10-12) Pneumonia accounts for as much as $50 \%$ of septic shock cases in cancer patients. 
Febrile neutropenia is one of the most frequent complications in cancer patients and sometimes, if not promptly treated has an unfavourable prognosis with evolution towards serious clinical problems such as septic shock, acute organ dysfunction, disseminated intravascular coagulation and eventually death. The lungs are one of the most frequent sites of infection in oncological patients during neutropenia. (10-13)

Diagnostic exams such as CXR and CT are required in febrile cancer patient. However, CXR, especially during neutropenia, is not very specific. It is unable to make differential diagnosis between bacterial, viral or mycotic pneumonia, and for this reason, in the suspicion of a fungal etiology (Candida or Aspergillus) patients undergo chest CT scan,that has higher specificity. Moreover, CXR has low sensitivity if performed in the initial phase of infection, particularly if performed in single projection: on the first day of fever it is often negative. $(14,15)$ The literature has also already established the inconsistent role of CXR in the diagnosis of lung infection in the neutropenic patient probably due to the low number of neutrophils involved in the production of the inflammatory response. (13) Chest CT scan is complicated by organizational difficulties and greater exposure to ionizing radiation, especially in these patients already subjected to many radiological examinations. (16) For all these reasons we need new methods for a correct and quick diagnosis of pneumonia.

Despite the large literature in the pediatric field on the role of LUS in the diagnosis of pneumonia, to our knowledge there are no studies concerning its utilization in the diagnosis and follow-up of pulmonary infection in children with cancer.

In our Unit of Pediatric Hematology and Oncology we recently introduced the routinely use of bed-side LUS, as an aid to physical examination, in order to evaluate pulmonary involvement and subsequent clinical management in both febrile in- and out-patients. This technique has recently been included in the diagnostic management of our patients, to optimize diagnostic and therapeutic choices during the Covid-19 pandemic.

The goal of this study is to explore the use of LUS as a tool for the diagnosis of pneumonia in children with cancer, neutropenic and non neutropenic.

In detail the primary objective of our study is to evaluate the diagnostic accuracy of LUS in pediatric cancer patients. We want to evaluate if, during neutropenia, there is a reduction in sensitivity of the ultrasound, similar to the CRX, compared to the non-neutropenic patients or if it allows to obtain a faithful image, such as the chest CT scan. Furthermore, we want to evaluate whether the underlying disease, the therapies administered (chemotherapy and radiotherapy) and the state of neutropenia can be factors influencing the sensitivity and specificity of the ultrasound examination.

The secondary objectives of the study are to evaluate the role of LUS in the follow-up of patients with lung infection and the tolerability of LUS, taking into account child's age and his psycho-physical health.

\section{Methods and analysis}

This prospective observational study was performed at the Unit of Pediatric Hematology and Oncology in Catania between March and June 2020, at the beginning of the spread of the Covid-19 epidemic in Italy. It was used as a complementary technique to the physical examination, with the aim of limiting daily outpatient access or in-ward admission and transfer of patients to other departments to perform tests. All admitted cancer patients undergoing therapy (steroid, chemotherapy, radiotherapy) with suspected infection in progress, participated in the study. As a case-control, a similar number of patients, with no signs of infection, randomly picked, underwent LUS. The procedures performed were in accordance with the principles of the 1964 Declaration of Helsinki and its later amendments (2013). Informed consent was obtained from all participants.

The inclusion criteria were age 0-18 years, diagnosis of leukemia or solid tumor, therapy in progress. Patients with the following features were excluded: ongoing asthma crisis, cystic fibrosis, bronchodysplasia, congenital cardio-pulmonary malformations, primary and metastatic pleuro-pulmonary tumor localization.

Infection was defined as: body temperature $(\mathrm{T})$ greater than or equal to $38^{\circ} \mathrm{C}$ and increased c-reactive protein $(\mathrm{CRP})$ (normal range $0-5 \mathrm{mg} / \mathrm{dl}$ ) and/or procalcitonin (normal range $0-01 \mathrm{ng} / \mathrm{ml}$ ), with or without 
respiratory signs and symptoms (cough, tachydispnea, $\mathrm{SaO}_{2}<96 \%$, rales, reduction of vesicular murmur $(\mathrm{VM}))$.

For each patient we assessed age, gender, underlying cancer, the absolute number of white blood cells and neutrophils at the time of the LUS, distinguishing the patients in

- neutropenic, (neutrophils less than or equal to 1000/mmc)

- non-neutropenic (neutrophils greater than 1000/mmc).

We reported the presence or absence of fever considering as $\mathrm{T}$ greater than or equal to $38^{\circ} \mathrm{C}$, respiratory symptoms and signs, the results of hematological tests for infection. CXR and/or chest CT scans were also recorded.

CXR and chest CT were evaluated with radiologists and considered positive in the presence of pulmonary thickening or marked accentuation of the bronchovascular texture.

Chest CT scans were considered positive in the presence of pulmonary thickening or ground glass.

These tests were performed only if considered useful and appropriate for diagnostic purposes and clinical management.

LUS was always performed by two operators: a pediatrician with a six-month ultrasound training, and an expert sonographer pediatrician who reviewed all exams with a 5-10 MHz linear probe. The probe was placed perpendicularly, oblique and parallel to the ribs in the anterior, lateral and posterior thorax as described by Copetti \& Cattarossi with the patient supine and seated to scan the posterior thorax. (17) The sonographer was unaware of the CRX results.

Pneumonia was diagnosed in the presence of lung consolidation, air or fluid bronchograms in the sub-pleural region $>1 \mathrm{~cm}$, multiple air or fluid bronchograms, air bronchogram $<1 \mathrm{~cm}$ with multiple $\mathrm{B}$ lines in the neighboring sites, confluent B lines or white lung as previously classified. $(2,18,19)$

All ultrasound examinations represented by A-lines only, rare B-lines (less than 3 per ultrasound scan) or single and isolated aerial bronchogram $<1 \mathrm{~cm}$ were considered normal.

In cases of positive LUS a control ultrasound was repeated to evaluate the evolution of the described picture after 3 and 7 days. In cases with persistent positive LUS, a monthly sonography was performed.

The compliance of children during the ultrasound examination by assigning a score from 0 to 2 was also evaluated:

- 0 if he was uncooperative (if the patient cries or refuses to undergo the exam),

- 1 if he was indifferent during the exam,

- 2 if he was proactive (takes the exam as a game, participates curiously in the exam).

We divided the recruited patients into 4 groups:

1. non-infected non-neutropenic patients are patients with absolute number of neutrophils greater than or equal to $1000 / \mathrm{mmc}$ without fever and with normal value of CRP and/or procalcitonin,

2. non-infected neutropenic patients are patients with absolute number of neutrophils less than or equal to $1000 / \mathrm{mmc}$ without fever and with normal value of CRP and/or procalcitonin,

3. infected non-neutropenic patients are patients with absolute number of neutrophils greater than or equal to $1000 / \mathrm{mmc}$ with fever and with high level of CRP and/or procalcitonin,

4. infected neutropenic patients are patients with absolute number of neutrophils less than or equal to $1000 / \mathrm{mmc}$ with fever and with high level of CRP and/or procalcitonin, 
Patients of the first two groups did not show signs of ongoing infection and ultrasound results were analyzed in order to identify if there was an increase in false positives related to the underlying disease, the treatments administered for cancer or the number of white blood cells and neutrophils in patients without infection signs. Groups 3 and 4 include the cases with suspected infection.

LUS results in the third and fourth groups were analyzed with the aim of evaluating the sensitivity of LUS compared to CXR and CT images.

\section{Results}

We recruited 38 patients: 21 without signs of infection disease and 17 with signs of infection,23 (60\%) male and $15(40 \%)$ female. Diagnosis was acute leukemia (AL) in 22, brain tumor in 5, lymphoma in 4, sarcoma in 3 (1 renal sarcoma (RS),1 rhabdomyosarcoma (RMS), 1 osteosarcoma (OS)), Wilms tumor (WT) in 2, neuroblastoma (NBL) and desmoid fibromatosis in 1 patient. The mean age was 9.2 years and the median 9 years.

All patients underwent LUS, 16 underwent CXR, 3 chest CT.

Group 1 included 8 cases (21\%) of non-infected non-neutropenic patients, mean age 10.3 years, median 9.5 years.

The 8 LUS were negative. CXR was performed in 4 cases, as part of the initial evaluation at onset of cancer, and was negative in all cases. (Table 1)

Group 2 included were 13 (34\%) non-infected neutropenic patients, the mean age was 7.1 years, the median age 5 years; in only one case, ultrasonography revealed rare B lines and bilaterally subpleural air bronchograms; according to CXR images these findings were probably related to leukemic infiltrates present at the onset of the disease. In this group there was an agreement between ultrasound and CXR of $83 \%$. Particularly, the finding on x-ray of a nuanced hilar thickening, in a patient with onset of acute lymphoblastic leukemia, afebrile and with normal CRP, was not evidenced on LUS. (Table 1)

Group 3 recluted 7 infected non-neutropenic patients (19\%), mean age 11 years, median 9 years. Three patients $(42 \%)$ presented with respiratory symptoms; two initially asymptomatic patients subsequently presented respiratory symptoms. (Table 1 ) Of the seven LUS performed, 5 were positive and 2 were negative.

6 CXR were performed: 4 were negative and 2 were positive. In case 25 a second CXR was repeated after the onset of respiratory symptoms and turned out positive.

Between the ultrasound examinations and the radiographic examinations there was agreement in $67 \%$ of cases. In this group, 3 pathological chest CT scans were performed with $100 \%$ concordance LUS. In one of the three cases $(33 \%)$ chest CT was discordant with CXR.

LUS images were pathological, in the absence of initial respiratory symptoms, which appeared in the following 24 hours with a rapid decline in lung function in two cases. In these two cases (patient 25 and 26) the negativity of the radiographic examination can be considered as false negative, in fact the patients presented both pathological ultrasound images and afterwards clinical symptoms compatible with lung infection. In both cases, for clinical worsening and the onset of respiratory symptoms, CXR (patient 25), repeated 4 days later, and chest CT (patient 26) performed one day after the CRX execution, were positive for pneumonia. (Fig. 1)

Group 4 included 10 infected neutropenic patients (26\%), mean age 8.5 years, median 10 years. We found respiratory symptoms only in 1 case. The results of laboratory and instrumental tests are shown in Table 2.

In $50 \%$ of cases the LUS was found to be pathological.

None of the 10 patients underwent a CXR and in no case was it necessary to perform a chest CT for the benign course of the infection. 
Between group 3 and 4, 10 patients presented positive LUS and underwent ultrasound follow-up at three and seven days from first LUS showing resolution of the pathological signs in 6 cases. In the 4 cases with persistent pathological images at day seven, a further LUS was performed after 1 month, with resolution of pneumonia in all cases.

\section{Statistical analysis and evaluation of diagnostic accuracy of LUS}

Statistical analysis showed that LUS specificity was $95 \%$, with only one finding of pathological ultrasound in an afebrile patient, in the absence of respiratory symptoms and normal inflammation indices. The images matched the CXR ones and were interpreted as leukemic infiltrates present at the onset of the disease.

CXR, performed on 16 patients, showed a specificity of $80 \%$ displaying pathological images in two cases of onset of leukemia, in apyretic and asymptomatic patients (non-infected patients), likely correlated to infiltration by leukemic cells.

Sensitivity of LUS for the diagnosis of pulmonary infections was 100\%. In all cases with specific symptomatology and/or CXR or CT images compatible with pneumonia, LUS was positive. Five febrile neutropenic patients in whom there were no respiratory symptoms or comparative radiological tests, all resulted negative at LUS, were excluded from the analysis because we did not know if these exams were true or false negatives. Probably these febrile events indicated an infection without pulmonary involvement.

The sensitivity of CXR was 50\%. The positive predictive value of LUS was $90 \%$ while that of CXR was $50 \%$.

The negative predictive value of LUS was $100 \%$ while that of CXR was $80 \%$.

Pediatric compliance during ultrasound examination:

Seven patients (18.4\%) obtained a score of 0 . These children, all younger than 6 years old, were afraid to undergo the ultrasound examination, but the ultrasound was nevertheless completed with valid results. A score of 1 was assigned to 11 patients (28.9\%), aged 6-17 years; they faced the diagnostic exam with indifference. Finally, 20 children (52.6\%) aged 2-16 years showed interest and curiosity during the ultrasound exam, obtaining a score of 2. (Table 3 )

Most patients with score 2 had received tumor diagnosis in a previous admission, while the ones not cooperative or wary were at the onset of their disease. There was no statistically significant difference between febrile and apyretic patients.

\section{Discussion}

Although for a long time it was thought that LUS was not feasible due to the air content, numerous studies in adults and subsequently in children, showed its efficacy for the diagnosis of pneumonia with sensitivity and specificity superior to CXR. $(17,19-24)$ Therefore, LUS is now identified as a valid substitute for CXR in the course of respiratory tract infections in children with the aim of reducing exposure to ionizing radiation. Moreover, CXR does not allow localization of infection in cancer patients in over $44 \%$ of cases. (13) As demonstrated by Gerristen et al., the sensitivity of CT at low doses of radiation is $73 \%$ performed on the first day of febrile neutropenia versus the sensitivity of 36\% of CXR. (13) Heussel et al. showed that in more than $50 \%$ of febrile neutropenic patients with normal CXR, there were signs of pulmonary inflammation on CT. (25)

Ultrasonography is the ideal tool for its speed, non-invasiveness, easy repeatability and simple interpretation of the examination after appropriate training even by non-radiology specialists. This last feature is of increasing importance in relation to the concept of personalized medicine. (5) The "bed side" ultrasonography, performed in the emergency departments or in critically patients, allows a more detailed evaluation of the individual patient obtaining an ever more patient-based rather than disease-based care approach. (5)

If in pediatric clinical practice, LUS is becoming an increasingly useful examination; howevwer, no study about its accuracy has ever been performed on the pediatric cancer population, where factors such as type 
of neoplasia, chemotherapy and thoracic radiotherapy with its pulmonary toxic effects and neutropenia may reduce the specificity and sensitivity of the tests.

Our data indicated that the underlying cancer, the administered therapies, the number of neutrophils did not influence the result, and the specificity was $95 \%$, comparable to that described in the pediatric population for the diagnosis of pneumonia.(26) The sensitivity, even if calculated in a small series, was $100 \%$.

Our data, with the limit of a small sample, showed that in non-neutropenic febrile patients, LUS has an important role, comparable with the literature on pneumonia cases in pediatric age. (26-30) In this population, LUS showed better diagnostic accuracy than CXR, which had a specificity of $80 \%$, and a sensitivity of $50 \%$, considerably lower than literature data on pediatric population but similar to adult cancer population. (13) LUS showed to have early positivization, even before the onset of clinical respiratory symptoms. This test could therefore be used as a screening tool for pulmonary infections in febrile cancer patients, in order to undertake early specific and targeted treatments or to direct the diagnostic-therapeutic work-up. Moreover, a LUS follow-up allowed to modify or to stop the anti-infection treatment according to the evolution of the process.

In febrile neutropenic patients, despite the absence of respiratory symptoms, it was possible to find lung lesions on ultrasound in $50 \%$ of cases, indicating that this method can be an aid technique for the diagnosis of infections during neutropenia, while CXR, with its low sensitivity is not indicated in the suspicion of lung infection during neutropenia. Five out of 10 patients presented a positive LUS for pneumonia. These data show that in $50 \%$ of patients with febrile neutropenia there is an ongoing lung infection; the latter often resolves thanks to empirical broad-spectrum antibiotic therapy but in a smaller percentage of cases it can evolve towards acute respiratory failure or sepsis. (31) Likely the reason why LUS has greater sensitivity than CXR in this population can be explained by the results obtained by Shah VP et al. (32) This study demonstrated that while for pulmonary thickenings of size greater than $1 \mathrm{~cm}$ the CXR and the LUS have a similar diagnostic accuracy, in pulmonary thickenings smaller than $1 \mathrm{~cm}$ LUS has a clearly superior sensitivity. Therefore, it can be hypothesized that in the patient with a low number of neutrophils and consequent deficient inflammatory response, the infection predisposes to develop subcentimetric thickening, not detectable on CXR. This hypothesis is confirmed by the few data we collected: in 4 out of 5 febrile neutropenic patients $(80 \%)$, pulmonary thickening on LUS were subcentimetric. This finding could open the door to a new simple diagnostic tool to be used in all neutropenic febrile cancer patients.

Most patients were compliant to LUS, demonstrating the low invasiveness of the method even in this population. The less cooperative patients were younger than 6 year or patients at the onset of cancer, often frightened by many diagnostic procedures (bone marrow aspirate, lumbar puncture, placement of central venous catheters or excisional biopsy), the hospital environment and the disease itself. Moreover, LUS was efficaciously performed, without the need of sedation, what is not always feasible for CXR or CT.

The possibility of using a tool, such as LUS, which is minimally invasive, repeatable, bedside suitable, radiation free and, at the same time, accurate, as a screening for the early detection of lung infection and monitoring its evolution, can have a great impact on the management and outcome of the pediatric cancer patient.

We hypothesize a role for LUS, as a first imaging technique, and propose a possible diagnostic work-up flow chart that includes LUS for the management of patients with signs of infection, with the aim of reducing radiation exposure and inserting a non-invasive and bed side examination (Fig. 2). Obviously, the proposed flow chart does not exempt from individual evaluation and specific clinical management.

\section{Conclusion}

In conclusion, LUS seems to be an accurate and well tolerated method in the diagnosis of pneumonia in febrile neutropenic and non-neutropenic pediatric cancer patients. We therefore believe in the potential of this method for the diagnosis and follow-up of lung infections in pediatric cancer patients. Further studies and larger case series are needed to confirm the hypothesis put forward with this pilot study. 
Acknowledgment: The medical staff of the Pediatric and Pediatric Emergency Room Department of the Cannizzaro Emergency Hospital, Catania for the training performed in the execution of lung ultrasound

\section{References}

1. Joyner C, Herman RJ, Reid JM. Reflected ultrasound in the detection and localization of pleural effusion. JAMA 1967; 200: 399-402

2. Iorio G, Capasso M, De Luca G, Prisco S, Mancusi C, Laganà B, Comune V. Lung Ultrasound in the Diagnosis of Pneumonia in Children: Proposal for a New Diagnostic Algorithm. Peer J 2015 Nov 10;3:e1374. doi: $10.7717 /$ peerj.1374

3. Urbankowska E, Krenke K, Drobczynsk L, Korczynski P, Urbankowski T, Krawiec M, Kraj G Brzewski M, Kulus M. Lung ultrasound in the diagnosis and monitoring of community acquired pneumonia in children. Respiratory Medicine 109. (2015), 1207-1212

4. Balk DS, Lee C, Schafer J, Welwarth J, Hardin J, Novack V, Yarza S,Hoffmann B.Lung ultrasound compared to chest-X-ray for diagnosis of pediatric pneumonia: A meta-analysis, Pediatr Pulmonol 2018 Aug;53(8):1130-1139DOI: 10.1002/ppul.24020

5. Stadler J AM, Andronikou S, Zar H J. Lung Ultrasound for the Diagnosis of Community-Acquired Pneumonia in Children . Pediatr Radiol 2017 Oct;47(11):1412-1419.doi: 10.1007/s00247-017-3910-1.

6. Soldati G, Copetti R. Ecografia toracica. C.G. Edizioni Medico Scientifiche. II edizione. 2012

7. Lichtenstein DA, Meziere G, Lascols N, Biderman P, Courret JP, Gepner A, Goldstein I, TenoudjiCohen M: Ultrasound diagnosis of occult pneumothorax. Crit Care Med 2005; 33: 1231-8

8. Cox M, Soudack M, Podberesky D. J., Epelman M.. Pediatric chest ultrasound: a practical approach. Pediatr Radiol (2017) 47:1058-1068

9. Riccabona M. Ultrasound of the chest in children (mediastinum excluded). Eur Radiol 2008; 18:390399.

10. Hae Jin Kim, MD So Young Park, MD, Ho Yun Lee, MD Kyung Soo Lee, MD Kyung Eun Shin, MD Jung Won Moon. Ultra-Low-Dose Chest CT in Patients with Neutropenic Fever and Hematologic Malignancy: Image Quality and Its Diagnostic Performance. Cancer Res Treat. 2014;46(4):393-402

11. Maschmeyer G, Link H, Hiddemann W, Meyer P, Helmerking M, Eisenmann E, et al. Pulmonary infiltrations in febrile patients with neutropenia: risk factors and outcome under empirical antimicrobial therapy in a randomized multicenter study. Cancer. 1994;73:2296-304.

12. Rossini F, Verga M, Pioltelli P, Giltri G, Sancassani V, Pogliani EM, et al. Incidence and outcome of pneumonia in patients with acute leukemia receiving first induction therapy with anthracyclinecontaining regimens. Haematologica. 2000;85:1255-60

13. Gerritens M G, Willemink M J , Pompe E, van der Bruggen T, van Rhenen A, Lammers J W J , Wessels F, Sprengers R W, de Jong P A , Minnema M C. Improving Early Diagnosis of Pulmonary Infections in Patients With Febrile Neutropenia Using Low-Dose Chest Computed Tomography , PLoS One 2017. Feb24;12(2):e0172256. doi:10.1371/journal.pone.0172256. eCollection 2017

14. Donowitz GR, Harman C, Pope T, Stewart FM. The role of the chest roentgenogram in febrile neutropenic patients. Arch Intern Med. 1991;151:701-4

15. Korones DN, Hussong MR, Gullace MA. Routine chest radiography of children with cancer hospitalized for fever and neutropenia: is it really necessary? Cancer. 1997;80:1160-4.

16. Stanzani M, Battista G, Sassi C, Lewis RE, Tolomelli G, Clissa C, et al. Computed tomographic pulmonary angiography for diagnosis of invasive mold diseases in patients with hematological malignancies. Clin Infect Dis. 2012;54:610-6

17. Coppetti R., Cattarossi L. Ultrasound diagnosis of pneumonia in children. Radiol Med, 2008 Mar;113(2):190-8. doi: 10.1007/s11547-008-0247-8. Epub 2008 Apr 2.

18. Volpicelli G, Elbarbary M, Blaivas M, et al; International Liaison Committee on Lung Ultrasound (ILC-LUS) for the International Consensus Conference on Lung Ultrasound (ICCLUS). International evidence-based recommendations for point-of-care lung ultrasound. Intensive Care Med 2012;38:577-91.

19. Reissig A, Gramegna A, Aliberti S; The role of lung ultrasound in the diagnosis and follow-up of community-acquired pneumonia. Eur J Intern Med 2012 Jul;23(5):391-7. 
doi:10.1016/j.ejim.2012.01.003. Epub 2012 Feb 21

20. Parlamento S., Copetti R., Di Bartolomeo S.; Evaluation of lung ultrasound for the diagnosis of pneumonia in the ED Am J Emerg Med 2009 May;27(4):379-84. doi: 10.1016/j.ajem.2008.03.009

21. Lichtenstein D, Goldstein I, Mourgeon E, Cluzel P, Grenier P, Rouby JJ. Comparative diagnostic performances of auscultation, chest radiography, and lung ultrasonography in acute respiratory distress syndrome. Anesthesiology. 2004; 100: 9-15

22. Iuri D, De Candia A, Bazzocchi M; Evaluation of the lung in children with suspected pneumonia: usefulness of ultrasonography. Radiol Med 2009 Mar;114(2):321-30. doi: 10.1007/s11547-008-0336-8. Epub 2008 Oct 25

23. Caiulo V. A., Gargani L., Caiulo S., Fisicaro F., Moramarco F., Latini G., Picano E., Mele G., The role of ultrasound in community-acquired pneumonia Pediatr Pulmonol 2013 Oct;48(10):1043-4. doi: 10.1002/ppul.22787. Epub 2013 Feb 8

24. Orso D., Ban A., Guglielmo N.; Lung ultrasound in diagnosing pneumonia in childhood: a systematic review and meta-analysis J Ultrasound 2018 Sep;21(3):183-195. doi: 10.1007/s40477-018-0306-5. Epub 2018 Jun 21

25. Heussel CP, Kauczor HU, Heussel GE, Fischer B, Begrich M, Mildenberger P, et al. Pneumonia in febrile neutropenic patients and in bone marrow and blood stem-cell transplant recipients: use of high-resolution computed tomography. J Clin Oncol. 1999;17:796-805

26. Pereda M. A, Chavez M. A., Hooper-Miele C. C., Gilman R. H., Steinhoff M. C., Ellington L. E., Gross M., Price C. , Tielsch J. M., Checkley W Lung ultrasound for the diagnosis of pneumonia in children: a meta-analysis. Pediatrics 2015 Apr;135(4):714-22. doi: 10.1542/peds.2014-2833. Epub 2015 Mar 16

27. Xin H, Li J, Hu HY. Is Lung Ultrasound Useful for Diagnosing Pneumonia in Children?: A MetaAnalysis and Systematic Review. Ultrasound Q 2018 Mar;34(1):3-10.doi: 10.1097

28. Hendaus M A, Jomha F A, Alhammadi A H. Lung ultrasound for the diagnosis of childhood pneumonia: a safe and accurate imaging mode. Ther Clin Risk Manag. 2015 Dec 9;11:1817-8. doi: 10.2147/TCRM.S96222. eCollection 2015.

29. Urbankowska E, Krenke K, Drobczyński L, Korczyński P, Urbankowski T, Krawiec M, Kraj G, Brzewski $\mathrm{M}$, Kulus MLung ultrasound in the diagnosis and monitoring of community acquired pneumonia in children. Respir Med 2015 Sep;109(9):1207-12. doi:10.1016/j.rmed.2015.06.011. Epub 2015 Jun 23.

30. Jaworska J, Komorowska-Piotrowska A, Pomiećko A, Wiśniewski J, Woźniak M, Littwin B, Kryger M, Kwaśniewicz P, Szczyrski J, Kulińska-Szukalska K, Buda N, Zbigniew Doniec, Kosiak W. Consensus on the Application of Lung Ultrasound in Pneumonia and Bronchiolitis in Children. Diagnostics (Basel). 2020 Nov; 10(11): 935.

31. Santolaya M. E., Alvarez A. M., Aviles C. L., Becker A., King A., Mosso C., O'Ryan M., Paya E., Salgado C., Silva P., Topelberg S., Tordecilla J, Varas M., Villarroel M., Viviani T, Zubieta M. Predictors of severe sepsis not clinically apparent during the first twenty-four hours of hospitalization in children with cancer, neutropenia, and fever: a prospective, multicenter trial. Pediatr Infect Dis J 2008 Jun;27(6):53843. doi:10.1097/INF.0b013e3181673c3c

32. Vaishali P Shah 1, Michael G Tunik, James W Tsung Prospective Evaluation of Point-Of-Care Ultrasonography for the Diagnosis of Pneumonia in Children and Young Adults JAMA Pediat 2013 Feb;167(2):119-25. doi: 10.1001/2013.jamapediatrics.107

\section{Hosted file}

Table 1 pediatric pulmonology.docx available at https://authorea.com/users/344797/articles/ 524885-pleuro-pulmonary-ultrasound-in-the-diagnosis-and-follow-up-of-lung-infections-inchildren-with-cancer-a-pilot-sudy

\section{Hosted file}

tab 2 pediatric pulmonology.docx available at https://authorea.com/users/344797/articles/ 524885-pleuro-pulmonary-ultrasound-in-the-diagnosis-and-follow-up-of-lung-infections-in- 
children-with-cancer-a-pilot-sudy

\section{Hosted file}

tab 3 pediatric pulmonology.docx available at https://authorea.com/users/344797/articles/ 524885-pleuro-pulmonary-ultrasound-in-the-diagnosis-and-follow-up-of-lung-infections-inchildren-with-cancer-a-pilot-sudy
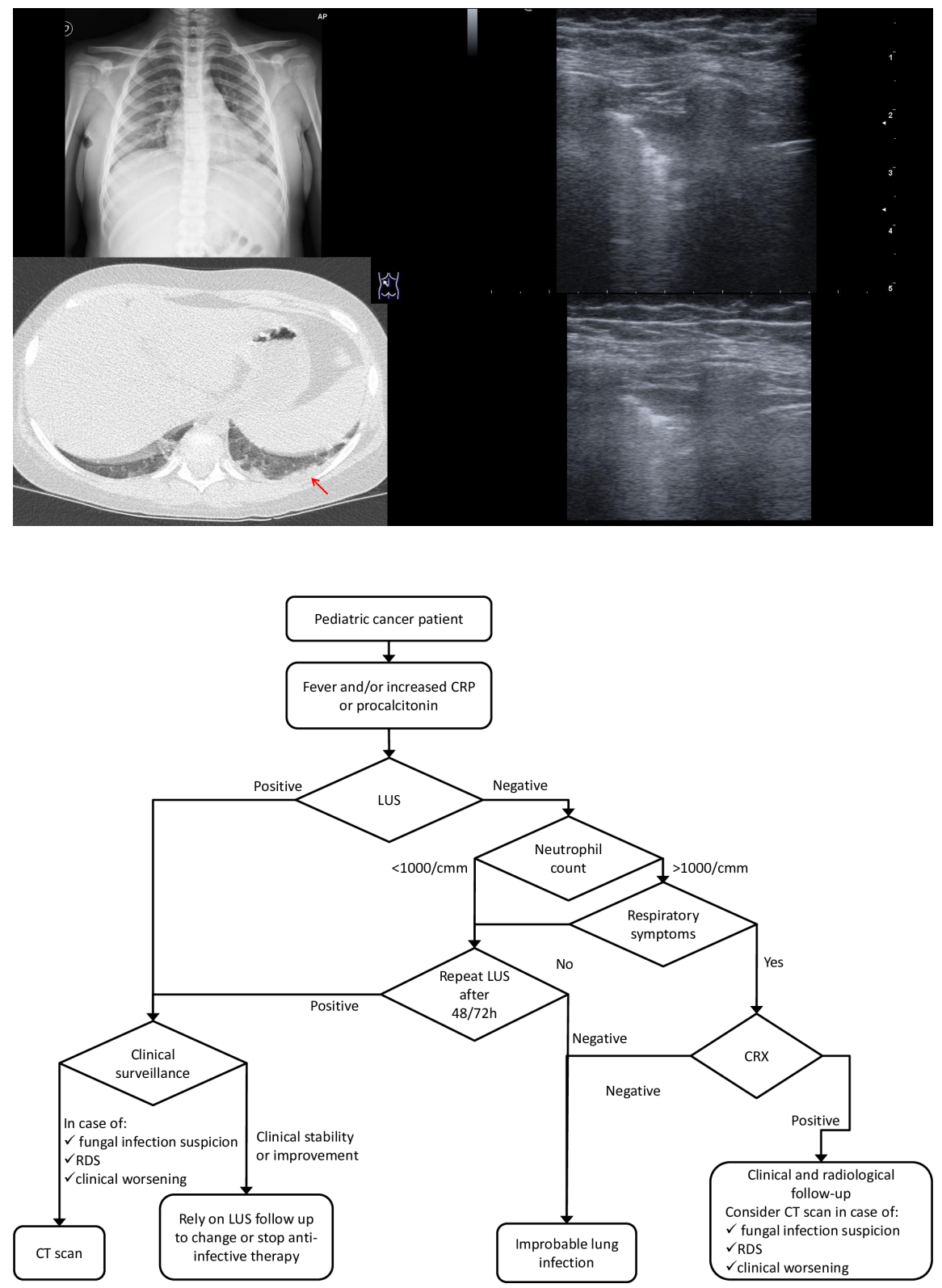